\title{
The Utilization of the Generating Function Technique in the Discovery of Solutions for the Three-Dimensional Navier-Stokes Equation System
}

\author{
Robert Jackson, Stilwell, Kansas
}

\begin{abstract}
The derivation of solutions to the Navier-Stokes (system of) equations (NSEs), in three spatial dimensions, has been an enigma for as time can tell. This study wishes to show how to eradicate this problem via the usage of a recently proposed method for solving partial differential equations called the Generating Function Technique, or GFT for short. The paper will first quickly define the NSEs with and without an external force, then provide a quick synopsis of the GFT. Next, the study will derive solutions to these two major problems and give an analysis of the data concerning a specific set of criteria established by the Clay Mathematics Institute to determine the smoothness and existence of solutions. Finally, the paper will provide a brief discussion of the results.
\end{abstract}

\section{Introduction.}

The motion of viscous fluids is depicted through Euler and Navier-Stokes equations (NSEs) [1]. The equations come from applying Newton's second law of motion to viscous fluids [2]. The comparisons are significant in many fields associated with physics.

Mathematicians considered the NSEs attractive since they possess many unresolved features. For instance, the Clay Mathematics Institute created a one-million-dollar prize if an individual can prove some of the elements in the very early parts of the twentieth century [1]. The educational organization wanted to know whether solutions existed for certain conditions (i.e., the kinetic energy of a fluid substance should never exceed a particular value). Also, it wanted to know if these solutions maintained their smoothness and continuous nature at all points under an infinite number of differentiations. In other words, were the solutions $\overline{C^{\infty}}$ functions. 
The purpose of this study is to: 1.) find solutions to the NSEs with or without an external force; and 2.) see if the exact solutions satisfied some of the criteria established by the Clay Mathematics Institute. The generating function technique (GFT) is used in finding exact solutions to the NSEs.

2. A short synopsis on the NSEs and its relevant vector fields.

The NSEs are a group of nonlinear PDEs involving vector fields [1,3]. The three equations are defined as follows:

$$
(\vec{v}(x, y, z, t) . \nabla) \vec{v}(x, y, z, t)+\partial_{t} \vec{v}(x, y, z, t)=\vec{f}(x, y, z, t)-\frac{\nabla p(x, y, z, t)}{\rho}+v \Delta \vec{v}(x, y, z, t) \quad\left(\quad \overline{(x, y, z) \in \mathbb{R}^{3}, t \geq[0, \infty)}\right),
$$

and

$$
\begin{aligned}
& \nabla \cdot \vec{v}(x, y, z, t)=0 \quad\left(\overline{(x, y, z) \in \mathbb{R}^{3}, t \geq[0, \infty)}\right),
\end{aligned}
$$

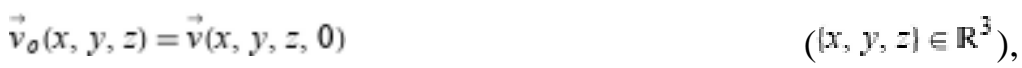

where vector field $\vec{v}$ is the velocity profile of the incompressible homogenous fluid, vector field $\vec{f}$ is an external force, function $p$ is the internal pressure, coefficient $\rho$ is the density of the fluid, and coefficient $v$ is the kinematic viscosity of the fluid. The two vector fields $\vec{v}_{\text {and }} \vec{f}$ are defined as:

$$
\vec{v}(x, y, z, t)=\left\{v_{x}(x, y, z, t), v_{y}(x, y, z, t), v_{z}(x, y, z, t)\right\},
$$

and

$$
\vec{f}(x, y, z, t)=\left\{f_{x}(x, y, z, t), f_{y}(x, y, z, t), f_{z}(x, y, z, t)\right\},
$$

respectively. Focus of the above equations will be limited to the following:

$$
\left|\vec{v}_{o}{ }^{\left(a_{1} a_{2} a_{3}\right)}(x, y, z)\right|=C^{\mathrm{K} a_{1} a_{2} a_{3}}\left(\sqrt{x^{2}+y^{2}+z^{2}}+1\right)^{-\mathrm{K}},
$$

and

$$
\left|\vec{f}^{\left(a_{1} a_{2} a_{3} m\right)}(x, y, z, t)\right|=C^{\mathrm{mK} a_{1} a_{2} a_{3}}\left(t+\sqrt{x^{2}+y^{2}+z^{2}}+1\right)^{-\mathrm{K}} .
$$

\section{Application of GFT.}

GFT is a method for solving [non]linear PDEs via the usage of a general solution $u_{g}$ that is comprised of [truncated] Laurent series sets of combinatorial number or trigonometric-based generating 
functions [4]. First, it requires an individual to determine the maximal and minimal power degree, $p_{s}$, through which the Laurent series is cut short. Then, one must solve a linear auxiliary/characteristic ordinary differential equation to obtain a function $\phi$ that is used in the transformed general solution $U_{g}$ for the principle nonlinear PDE that is in question. The transformed general solution $U_{g}$ can take the following form:

$$
U(\xi)=\sum_{i=1}^{2} \sum_{j=-p_{s}}^{p_{s}}\left(a_{\mathrm{ij}}\left(\sum_{k=0}^{\infty} 2 S_{k}(0)^{i} \phi(\xi)^{k}\right)^{j}+b_{\mathrm{ij}}\left(\sum_{k=0}^{\infty} 2 C_{k}(0)^{i} \phi(\xi)^{k}\right)^{j}\right),
$$

where the expression $S_{k}(0)$ is the square root of the $k$-th Fibonacci number at/about zero, or

$$
S_{k}(0)=\sin \left(\frac{\pi k}{2}\right),
$$

the expression $C_{k}(0)$ is the $k$-th Chebyshev $\mathrm{U}$ number at/about zero, or

$$
C_{k}(0)=\cos \left(\frac{\pi k}{2}\right),
$$

and the transformed variable $\xi$ for a $(3+1)$ [nonlinear] PDE is the transformed is simply defined as:

$$
\xi=\alpha t+\beta_{1} x+\beta_{2} y+\beta_{3} z
$$

Once the transformed general solution is established for the principle [nonlinear] PDE, the individual must transform the principle equation via ansatz and plug in the transformed general solution $U_{g}$. Using [exponential] terms found in the auxiliary solution $\phi$, one can define a set of algebraic equations that are necessary for solving constants like $a_{i j}, b_{i j}, \alpha, \beta_{1}, \beta_{2}$ and $\beta_{3}$ whenever possible. The transformed general solution $U_{g}$ becomes the exact solution $u_{e}$ to the principle [nonlinear] PDE after plugging in the constants described in the prior sentence.

4. Finding, then describing the solutions to the NSEs (via GFT).

Mathematica ${ }^{\circledR}$ was used to derive solutions to the NSEs with and without an external force. Mathematica ${ }^{\circledR}$ spreadsheets for the two sets of problems were provided in the supplementary data of this study.

4.1. Derivation of solutions for the NSEs with and without an external force.

Proposition 4.1.1 The general solution $u_{g}$ of the GFT can be used to derive putative exact solutions of the velocity vector field $\vec{v}$ and internal pressure p to NSEs with and without an external force $\vec{f}$. 
First, an individual must solve a particular order homogeneous auxiliary/characteristic ordinary differential equation like the following:

$$
\overline{\phi^{\prime \prime}(\xi)-\phi(\xi)=0}
$$

whose solution $\phi$ is given as:

$$
\phi(\xi)=c_{1} e^{\xi}+c_{2} e^{-\xi} .
$$

Next, one determines the maximal and minimal power for truncation $p_{s}$, which is equal to the value of 1 . Then using equation (3.1), (s)he establishes the transformed general solutions for the internal pressure $P$ and the vector fields $\vec{V}$ and $\vec{F}$, respectively:

$$
\begin{gathered}
P(\xi)=\frac{e^{-\xi} c(1,-1)\left(-i e^{\xi}+c_{1} e^{2 \xi}+c_{2}\right)\left(i e^{\xi}+c_{1} e^{2 \xi}+c_{2}\right)}{2\left(c_{1} e^{2 \xi}+c_{2}\right)}-\frac{e^{-\xi} c(2,-1)\left(-e^{\xi}+c_{1} e^{2 \xi}+c_{2}\right)\left(e^{\xi}+c_{1} e^{2 \xi}+c_{2}\right)}{2\left(c_{1} e^{2 \xi}+c_{2}\right)}, \\
V_{x}(\xi)=\frac{e^{-\xi} \mathrm{a} 1(1,-1)\left(-i e^{\xi}+c_{1} e^{2 \xi}+c_{2}\right)\left(i e^{\xi}+c_{1} e^{2 \xi}+c_{2}\right)}{2\left(c_{1} e^{2 \xi}+c_{2}\right)}-\frac{e^{-\xi} \mathrm{a} 1(2,-1)\left(-e^{\xi}+c_{1} e^{2 \xi}+c_{2}\right)\left(e^{\xi}+c_{1} e^{2 \xi}+c_{2}\right)}{2\left(c_{1} e^{2 \xi}+c_{2}\right)}, \\
V_{y}(\xi)=\frac{e^{-\xi} \mathrm{a} 2(1,-1)\left(-i e^{\xi}+c_{1} e^{2 \xi}+c_{2}\right)\left(i \xi^{\xi}+c_{1} e^{2 \xi}+c_{2}\right)}{2\left(c_{1} e^{2 \xi}+c_{2}\right)}-\frac{e^{-\xi} \mathrm{a} 2(2,-1)\left(-e^{\xi}+c_{1} e^{2 \xi}+c_{2}\right)\left(e^{\xi}+c_{1} e^{2 \xi}+c_{2}\right)}{2\left(c_{1} e^{2 \xi}+c_{2}\right)}, \\
V_{z}(\xi)=\frac{e^{-\xi} \mathrm{a} 3(1,-1)\left(-i e^{\xi}+c_{1} e^{2 \xi}+c_{2}\right)\left(i e^{\xi}+c_{1} e^{2 \xi}+c_{2}\right)}{2\left(c_{1} e^{2 \xi}+c_{2}\right)}-\frac{e^{-\xi} \mathrm{a} 3(2,-1)\left(-e^{\xi}+c_{1} e^{2 \xi}+c_{2}\right)\left(\xi^{\xi}+c_{1} e^{2 \xi}+c_{2}\right)}{2\left(c_{1} e^{2 \xi}+c_{2}\right)}, \\
\overline{F_{x}(\xi)=e^{-\xi} \psi_{1},} \\
F_{y}(\xi)=e^{-\xi} \psi_{2},
\end{gathered}
$$

and

$$
\overline{F_{z}(\xi)=e^{-\xi} \psi_{3}} \text {. }
$$

Note: the index $j$ was limited to -1 , all $b_{i j}$ and $d_{i j}$ coefficients were set to the value of zero to limit the computational cost for deriving the putative exact solutions. Also, parenthesis was used for indexing some coefficient. Finally, the external force $\vec{f}$ involved a 3-dimensional Gaussian diffusion process.

The transformed NSEs with external force $\vec{f}_{\text {is defined as follows: }}$

$$
\begin{aligned}
& -F_{x}(\xi)+\frac{\beta_{1} P^{\prime}(\xi)}{\rho}+\alpha V_{x}^{\prime}(\xi)-\left(\beta_{1}^{2}+\beta_{2}^{2}+\beta_{3}^{2}\right) v V_{x}^{\prime \prime}(\xi)+\beta_{1} V_{x}(\xi) V_{x}^{\prime}(\xi)+\beta_{2} V_{y}(\xi) V_{x}^{\prime}(\xi)+\beta_{3} V_{z}(\xi) V_{x}^{\prime}(\xi)=0, \\
& -F_{y}(\xi)+\frac{\beta_{2} P^{\prime}(\xi)}{\rho}+\beta_{1} V_{x}(\xi) V_{y}^{\prime}(\xi)+\alpha V_{y}^{\prime}(\xi)-\left(\beta_{1}^{2}+\beta_{2}^{2}+\beta_{3}^{2}\right) v V_{y}^{\prime \prime}(\xi)+\beta_{2} V_{y}(\xi) V_{y}^{\prime}(\xi)+\beta_{3} V_{z}(\xi) V_{y}^{\prime}(\xi)=0, \\
& -F_{z}(\xi)+\frac{\beta_{3} P^{\prime}(\xi)}{\rho}+\beta_{1} V_{x}(\xi) V_{z}^{\prime}(\xi)+\beta_{2} V_{y}(\xi) V_{z}^{\prime}(\xi)+\alpha V_{z}^{\prime}(\xi)-\left(\beta_{1}^{2}+\beta_{2}^{2}+\beta_{3}^{2}\right) v V_{z}^{\prime \prime}(\xi)+\beta_{3} V_{z}(\xi) V_{z}^{\prime}(\xi)=0
\end{aligned}
$$

and 


$$
\overline{\beta_{1} V_{x}^{\prime}(\xi)+\beta_{2} V_{y}^{\prime}(\xi)+\beta_{3} V_{z}^{\prime}(\xi)=0 .} \text {. }
$$

After plugging in the transformed general solutions into the above equations, one extracts a total of thirtyseven algebraic equations associated with $\overline{\left\langle e^{\xi}\right\rangle}$. Then (s)he uses the algebraic equations to solve for constants whenever possible. One set of solved constants yields the following results for the velocity vector field $\vec{v}$ and internal pressure $p$ :

$$
\begin{aligned}
\vec{v}(x, y, z, t)=\left\{\frac{1}{2\left(\beta_{1}^{2}+\beta_{2}^{2}+\beta_{5}^{2}\right)^{2} v}\left(\beta_{2}^{2}\left(-\psi_{1}\right)+\beta_{1} \beta_{2} \psi_{2}+\beta_{3}\left(\beta_{1} \psi_{3}-\beta_{3} \psi_{1}\right)\right)\right. \\
\quad \exp \left(-\left(\beta_{1}^{2}+\beta_{2}^{2}+\beta_{3}^{2}\right) v t+\beta_{1}(-x)-\beta_{2} y-\beta_{3} z\right)\left(c_{1}^{2} \exp \left(2\left(\left(\beta_{1}^{2}+\beta_{2}^{2}+\beta_{3}^{2}\right) v t+\beta_{1} x+\beta_{2} y+\beta_{3} z\right)\right)+1\right), \\
-\frac{1}{2\left(\beta_{1}^{2}+\beta_{2}^{2}+\beta_{5}^{2}\right)^{2} v}\left(\beta_{1}^{2} \psi_{2}-\beta_{2} \beta_{1} \psi_{1}+\beta_{3}\left(\beta_{3} \psi_{2}-\beta_{2} \psi_{3}\right)\right) \exp \left(-\left(\beta_{1}^{2}+\beta_{2}^{2}+\beta_{3}^{2}\right) v t+\beta_{1}(-x)-\beta_{2} y-\beta_{3} z\right), \\
\quad\left(c_{1}^{2} \exp \left(2\left(\left(\beta_{1}^{2}+\beta_{2}^{2}+\beta_{3}^{2}\right) v t+\beta_{1} x+\beta_{2} y+\beta_{3} z\right)\right)+1\right),-\frac{1}{2\left(\beta_{1}^{2}+\beta_{2}^{2}+\beta_{3}^{2}\right)^{2} v}\left(\left(\beta_{1}^{2}+\beta_{2}^{2}\right) \psi_{3}-\beta_{3}\left(\beta_{1} \psi_{1}+\beta_{2} \psi_{2}\right)\right) \\
\left.\quad \exp \left(-\left(\beta_{1}^{2}+\beta_{2}^{2}+\beta_{3}^{2}\right) v t+\beta_{1}(-x)-\beta_{2} y-\beta_{3} z\right)\left(c_{1}^{2} \exp \left(2\left(\left(\beta_{1}^{2}+\beta_{2}^{2}+\beta_{3}^{2}\right) v t+\beta_{1} x+\beta_{2} y+\beta_{3} z\right)\right)+1\right)\right\},,
\end{aligned}
$$

and

$$
p(x, y, z, t)=-\frac{\rho\left(\beta_{1} \psi_{1}+\beta_{2} \psi_{2}+\beta_{3} \psi_{3}\right) \exp \left(-\left(\beta_{1}^{2}+\beta_{2}^{2}+\beta_{3}^{2}\right) v t+\beta_{1}(-x)-\beta_{2} y-\beta_{3} z\right)}{\beta_{1}^{2}+\beta_{2}^{2}+\beta_{3}^{2}} .
$$

The initial condition $\overrightarrow{\vec{v}_{o}(x, y, z)}$ can be derived from the putative exact solution of the velocity vector field $\vec{v}$ by setting time t equal to zero or one must consider the equation (2.3). The integration of the above solutions and vector field external force $\vec{f}$ is given in the supplementary material.

To produce solutions to the NSEs without an external force field, one performs the same steps but sets the vector field $\vec{f}$ and the transformed vector field $\vec{F}$ to $\{0,0,0\}$. (S)he still obtains thirty-seven algebraic equations associated with $\overline{\left\langle e^{\xi}\right\rangle}$. Then (s)he uses the algebraic equations to solve for constants whenever possible. One set of solved constants yields the following results for the velocity vector field $\vec{v}$ and internal pressure $p$ : 


$$
\begin{aligned}
& \vec{v}(x, y, z, t)=\left\{\frac{(\mathrm{a} 1(1,-1)+\mathrm{a} 1(2,-1)) \exp \left(x \sqrt{-\beta_{2}^{2}-\beta_{3}^{2}}+\alpha t+\beta_{2} y+\beta_{3} z\right)}{2 \mathrm{c}_{2}}+\right. \\
& \frac{1}{2} \mathrm{c}_{2}(\mathrm{a} 1(1,-1)-\mathrm{a} 1(2,-1)) \exp \left(x\left(-\sqrt{-\beta_{2}^{2}-\beta_{3}^{2}}\right)-\alpha t-\beta_{2} y-\beta_{3} z\right), \\
& \frac{(\mathrm{a} 1(1,-1)+\mathrm{a} 1(2,-1)) \exp \left(x \sqrt{-\beta_{2}-\beta_{3}^{2}}+\alpha t+\beta_{2} y+\beta_{3} z\right)}{2 \mathrm{c}_{2}}+ \\
& \frac{1}{2} \mathrm{c}_{2}(\mathrm{a} 1(1,-1)-\mathrm{a} 1(2,-1)) \exp \left(x\left(-\sqrt{-\beta_{2}^{2}-\beta_{3}^{2}}\right)-\alpha t-\beta_{2} y-\beta_{3} z\right), \\
& \frac{(\mathrm{a} 1(1,-1)+\mathrm{a} 1(2,-1)) \exp \left(x \sqrt{-\beta_{2}-\beta_{3}^{2}}+\alpha t+\beta_{2} y+\beta_{3} z\right)}{2 \mathrm{c}_{2}}+, \\
& \left.\frac{1}{2} \mathrm{c}_{2}(\mathrm{a} 1(1,-1)-\mathrm{a} 1(2,-1)) \exp \left(x\left(-\sqrt{-\beta_{2}^{2}-\beta_{3}^{2}}\right)-\alpha t-\beta_{2} y-\beta_{3} z\right)\right\},
\end{aligned}
$$

and

$$
\begin{aligned}
& p(x, y, z, t)=-\frac{1}{2 \mathrm{c}_{2} \sqrt{-\beta_{2}^{2}-\beta_{3}^{2}}} \alpha \rho \exp \left(x\left(-\sqrt{-\beta_{2}^{2}-\beta_{3}^{2}}\right)-\alpha t-\beta_{2} y-\beta_{3} z\right) \\
& \left(\mathrm{c}_{2}^{2}(\mathrm{a} 1(1,-1)-\mathrm{a} 1(2,-1))+(\mathrm{a} 1(1,-1)+\mathrm{a} 1(2,-1)) \exp \left(2\left(x \sqrt{-\beta_{2}^{2}-\beta_{3}^{2}}+\alpha t+\beta_{2} y+\beta_{3} z\right)\right)\right) .
\end{aligned}
$$

The initial condition $\overrightarrow{\vec{v}_{o}(x, y, z)}$ can be derived from the putative exact solution of the velocity vector field $\vec{v}$ by setting time t equal to zero or one must consider the equation (2.3). The integration of the above solutions is given in the supplementary material.

\subsection{The assessment of smoothness and the existence of both sets of solutions.}

Lemma 4.2.1 If $\{\vec{v}(x, y, z, t), p(x, y, z, t)\} \in C^{\infty}\left([0, \infty) \mathbb{R}^{3}\right)$ must be satisfied for there to be exact solutions, then all GFT derived velocity vector field $\vec{v}$ and internal pressure p for NSEs with and without external force $\vec{f}$ are possibly valid solutions.

To ascertain the smoothness of the solutions, one must determine if the solutions are $\overline{C^{\infty}}$ functions. A $C^{\infty}$ function is differentiable for all degrees of differentiation. Since this type of functions and all its derivatives lack corners, it is considered smooth. Also, this type of function is considered continuous due to it and all of its derivatives do not possess any abrupt/discontinuous jumps/drops in value to infinity in both directions. As one can see, both sets of velocity vector fields $\vec{v}$ and the internal pressures $p$ are linear combinations of $\overline{C^{\infty}}$ functions (i.e. $e^{ \pm a x}$ ) concerning time and 3D-space; therefore, they are likely $\overline{C^{\infty}}$ functions themselves. 
Lemma 4.2.2 If the kinetic energy for the system of NSEs solutions must be globally bound, then ONLY the GFT derived putative solutions of velocity vector field $\vec{v}$ and internal pressure p for NSEs without external force $\vec{f}$ are still valid.

To determine whether the set of solutions exists as defined by the Clay Mathematics Institute an individual must assess whether kinetic energy for a scenario is globally bound. This kinetic energy is less than or equal to some constant $E$ and is the right side of the following equation:

$$
\mathrm{E} \geq \int_{0}^{\infty} \int_{0}^{\infty} \int_{0}^{\infty}|\vec{v}(x, y, z, t)|^{2} d z d y d x,
$$

Where the magnitude of the velocity vector field is defined as:

$$
|\vec{v}(x, y, z, t)|=\sqrt{v_{x}(x, y, z, t)^{2}+v_{y}(x, y, z, t)^{2}+v_{z}(x, y, z, t)^{2}} .
$$

The kinetic energy for the NSEs without external force $\vec{f}$ is zero while the kinetic energy for the NSEs with external force $\vec{f}$ is either nonintegrable or can be represented by the following expression if the constant $c_{l}$ is set to zero:

$$
\mathrm{E} \neq \frac{\left(\beta_{1}^{2}\left(\psi_{2}^{2}+\psi_{3}^{2}\right)-2 \beta_{3} \beta_{1} \psi_{1} \psi_{3}+\beta_{3}^{2}\left(\psi_{1}^{2}+\psi_{2}^{2}\right)-2 \beta_{2} \psi_{2}\left(\beta_{1} \psi_{1}+\beta_{3} \psi_{3}\right)+\beta_{2}^{2}\left(\psi_{1}^{2}+\psi_{3}^{2}\right)\right) e^{-2\left(\beta_{1}^{2}+\beta_{2}^{2}+\beta_{3}^{2}\right) v t}}{32 \beta_{1} \beta_{2} \beta_{3}\left(\beta_{1}^{2}+\beta_{2}^{2}+\beta_{3}^{2}\right)^{3} v^{2}} .
$$

(Note: if the constants $\beta_{1}, \beta_{2}$, and $\beta_{3}$ are imaginary numbers, the right side of the above expression is likely to grow exponentially become greater than the constant $E$ at some point in time.) This data suggests the kinetic energy for the NSEs without external force $\vec{f}$ is globally bound. In contrast, the kinetic energy for the NSEs with external force $\vec{f}$ is NOT globally bound.

Theorem 4.2.3 Via Clay Mathematics Institute criteria, GFT successfully derived solutions for the velocity vector field $\vec{v}$ and internal pressure p for NSE without external force $\vec{f}$ while the method failed to generate valid solutions for the vector field $\vec{v}$ and internal pressure p for NSEs with external force $\vec{f}$.

\section{Conclusion}


If an external force is lacking in the NSEs, the velocity vector field $\vec{v}_{\text {and }}$ the internal pressure $p$ are both smooth and continuous or $\overline{C^{\infty}}$ functions. Also, the kinetic energy of the velocity profile for this system is zero, which is less than some constant $E$; thus, the kinetic energy is globally bound for the system. In other words, both the Millennium prize criteria are satisfied. On the other hand, if the NSEs do possess an external force, the velocity vector field $\vec{v}_{\text {and }}$ the internal pressure $p$ which are still both smooth and continuous, or $\vec{C}^{\infty}$ functions. However, the kinetic energy of the velocity profile for this system can eventually obtain values higher than some constant $E$; therefore, the kinetic energy for this system is not globally bound. This individual data would suggest that only one of the criteria established by the Millennium prize can be met for NSEs with an external force $\vec{f}$. In other words, via complementary logic, there are no velocity vector fields $\vec{v}$, under an external force $\vec{f}$, that can satisfy both criteria set by the Clay Mathematics Institute for such a system.

\section{References}

[1] Feffreman, C.L. (2017) "Existence and Smoothness of the Navier-Stokes Equation," www.claymath.org, Clay Mathematics Institute, March 27, 2017, retrieved 2017-04-02

[2] Acheson, D. J. (1990) Elementary Fluid Dynamics, Oxford Applied Mathematics and Computing Science Series, Oxford University Press, ISBN 978-0-19-859679-0

[3] Batchelor G K. (1967) An introduction to fluid dynamics, Cambridge, England: Cambridge University Press.

[4] Jackson, R. (2019) “A Possible Theory of Partial Differential Equations,” www.vixra.org 1910.0064 